Article

\title{
Field Deployable Fiber Bragg Grating Strain Patch for Long-Term Stable Health Monitoring Applications
}

\author{
Vivien Schukar*, Nadine Kusche, Gerhard Kalinka and Wolfgang Habel \\ BAM Federal Institute for Materials Research and Testing, Unter den Eichen 87, \\ D-12205 Berlin, Germany; E-Mails: nadine.kusche@bam.de (N.K.); \\ gerhard.kalinka@bam.de (G.K.); wolfgang.habel@bam.de (W.H.)
}

* Author to whom correspondence should be addressed; E-Mail: vivien.schukar@bam.de; Tel.: +49-30-8104-3609; Fax: +49-30-8104-1917.

Received: 2 November 2012; in revised form: 7 January 2013 / Accepted: 8 January 2013 / Published: 16 January 2013

\begin{abstract}
A fiber Bragg grating (FBG) strain patch specially adapted for long-term and high-strain applications has been developed and characterized. The design concept for the patch is based on a glass-fiber reinforced plastic (gfrp) carrier material. The developed concept for the FBG integration into the carrier material was derived from reliable integration procedure of FBG sensors into composite structures. The patches' temperature sensitivity, strain gauge factor, fiber-matrix interface adhesion and fatigue behavior were characterized. As a result, FBG strain patches with linear temperature and strain behavior, as well as excellent fatigue resistance, were developed and can be used as part of a monitoring system for advanced composite materials in aerospace structures or wind turbine power plants.
\end{abstract}

Keywords: fiber Bragg grating; patch; strain gauge factor; temperature sensitivity; push-out test; validation; fatigue behavior

\section{Introduction}

Over the past years, fiber Bragg gratings have been used as sensors for structural health monitoring and smart structure applications. Compared to conventional strain sensors like resistance strain gauges or extensometers, FBG sensors have several advantages. Small size, light weight, immunity to electromagnetic fields and lightning, capability to be multiplexed in a single optical fiber, material 
integratability, and the ability to withstand high dynamic loadings, make FBG strain sensors especially suitable for structure monitoring when performance accuracy and reliability are of principle interest.

FBG are optical wavelength filters written into an optical fiber by an UV laser. Using the phasemask technique, the index of refraction of the core of the fiber has been locally and periodically modified. From the light spectrum travelling in the fiber, a small spectral band will be reflected at the grating, while the rest of the light will be transmitted. The Bragg condition defines the centre wavelength $\lambda_{B}$ of the small reflected band [1],

$$
\lambda_{\mathrm{B}}=2 n_{\mathrm{eff}} \Lambda
$$

with $n_{\text {eff }}$ effective index of refraction and $\Lambda$ grating period. FBG are both sensitive to strain and temperature. The shift in Bragg wavelength due to strain and temperature is given by

$$
\Delta \lambda_{B}=2\left(\Lambda \frac{\partial n_{\text {eff }}}{\partial l}+n_{\text {eff }} \frac{\partial \Lambda}{\partial l}\right) \Delta l+2\left(\Lambda \frac{\partial n_{\text {eff }}}{\partial T}+n_{\text {eff }} \frac{\partial \Lambda}{\partial T}\right) \Delta T
$$

with $\Delta l$ change in grating length, and $\Delta T$ change in temperature. A major advantage of FBG sensors is, additionally, that the sensor signal is encoded in the frequency domain and therefore is not affected by signal power fluctuations.

FBG sensors in bare optical fibers are very brittle and, therefore, difficult to handle, especially for untrained users. Subsequently, producers offer FBGs integrated or applied to a carrier material in order to simplify the handling and installation of the sensors. These optical strain gauges are also named "patches." Patches are available from different manufacturers and, depending on the patch design, their characteristics are different. In previous investigations [2], we compared the performance of several commercially available patches to their specifications in the datasheets. Although an international guideline for the use and testing of FBG-based optical strain sensors [3], exists and manufacturers are strongly recommended to use it, our experimental investigations showed deviations in the patch performance compared to the specified characteristics by the manufacturer. Additionally, we observed some of the surface-applied patches delaminated from the specimen during alternate bending tests at a flexural strain of $4 \mathrm{~mm} / \mathrm{m}$ at a frequency of $0.5 \mathrm{~Hz}$, as shown in Figure 1.

Figure 1. Alternate bending test with FBG-based patches. The optical signal of patch 1 decays at a flexural strain of $4 \mathrm{~mm} / \mathrm{m}$. The patch started to delaminate from the specimen.

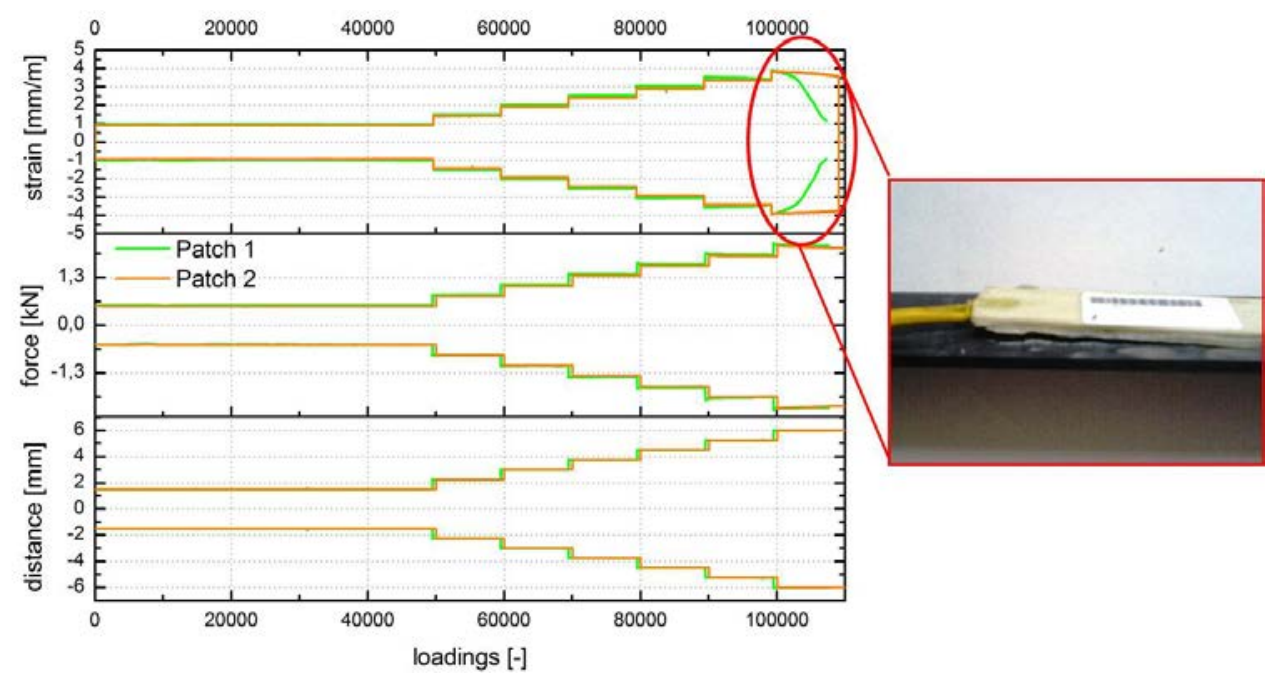


For this reason, it was necessary to develop a FBG patch with reliable and long-term stable mechanical and spectral properties. However, a carrier material glass-fiber-reinforced plastic (gfrp) was chosen so that the manufacturing technique could be easily adapted for the integration of optical fibers into composite structures.

\section{Sensor Design}

The patch was carefully designed. First, a gfrp carrier made of six layers of glass fabric $\left(80 \mathrm{~g} / \mathrm{m}^{2}\right)$ was laminated and vacuum-cured at $60{ }^{\circ} \mathrm{C}$. Patch carrier of size $50 \mathrm{~mm} \times 15 \mathrm{~mm} \times 0.6 \mathrm{~mm}$ were cut out of the gfrp carrier. Second, the optical fiber was prepared and attached to the surface of the patch carrier. Therefore, ptfe tubes are used to protect the fiber going over the sharp edges of the patch carrier. The tubes have to be placed carefully at the end of the sensitive area of the Bragg grating. Then, they are fixed on the carrier and sealed so that no liquid resin flows along the optical fiber. This way, the fiber is protected from becoming extremely brittle after hardening due to the resin flow. On top of the fiber that is fixed on the carrier, a final layer glass fabric was laminated and vacuum-cured to avoid mechanical damage of the fiber. In Figure 2, the developed sensor patch applied to a cfrp specimen is shown.

Because of the curing temperature of $60{ }^{\circ} \mathrm{C}$, the patch can only be used up to this temperature. For use at higher temperatures, the patch has to be post-cured at the desired temperature.

Figure 2. Sensor patch applied to cfrp specimen.

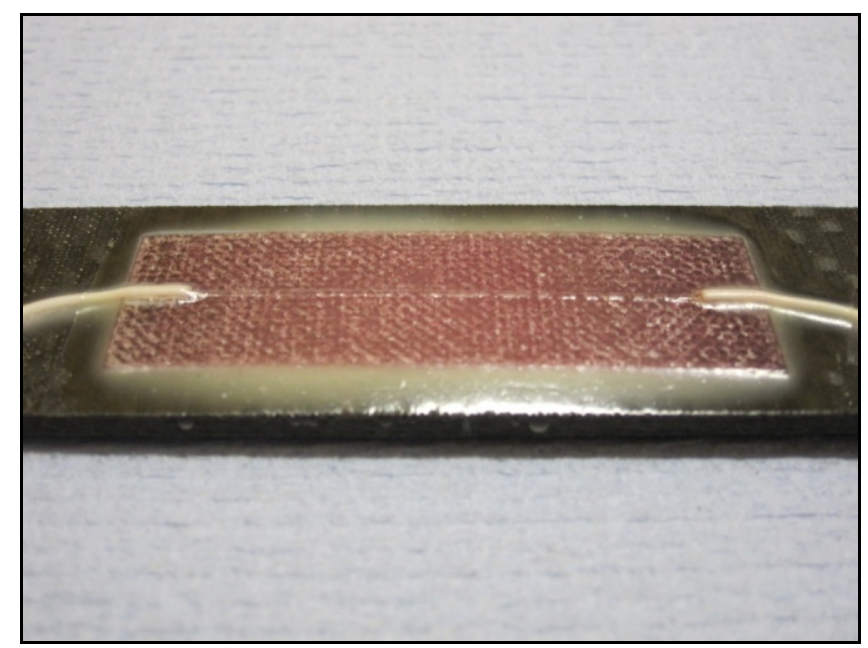

\subsection{Curing Process}

The FBG, embedded in the patch material, can additionally be used to monitor the patch curing process. What happens during the curing process in terms of residual stress and exothermal reaction is extremely important for the configuration of the FBG inside the patch. It is well known that the gfrp material shrinks significantly during curing, up to $2 \%$, depending on the resin system used. The resin curing process has been discussed in detail in [4].

The characteristic curing cycling can be observed with FBG sensors during embedment into the patch carrier, as shown in Figure 3. 
Figure 3. Cure cycling of a patch monitored with embedded FBG sensor.

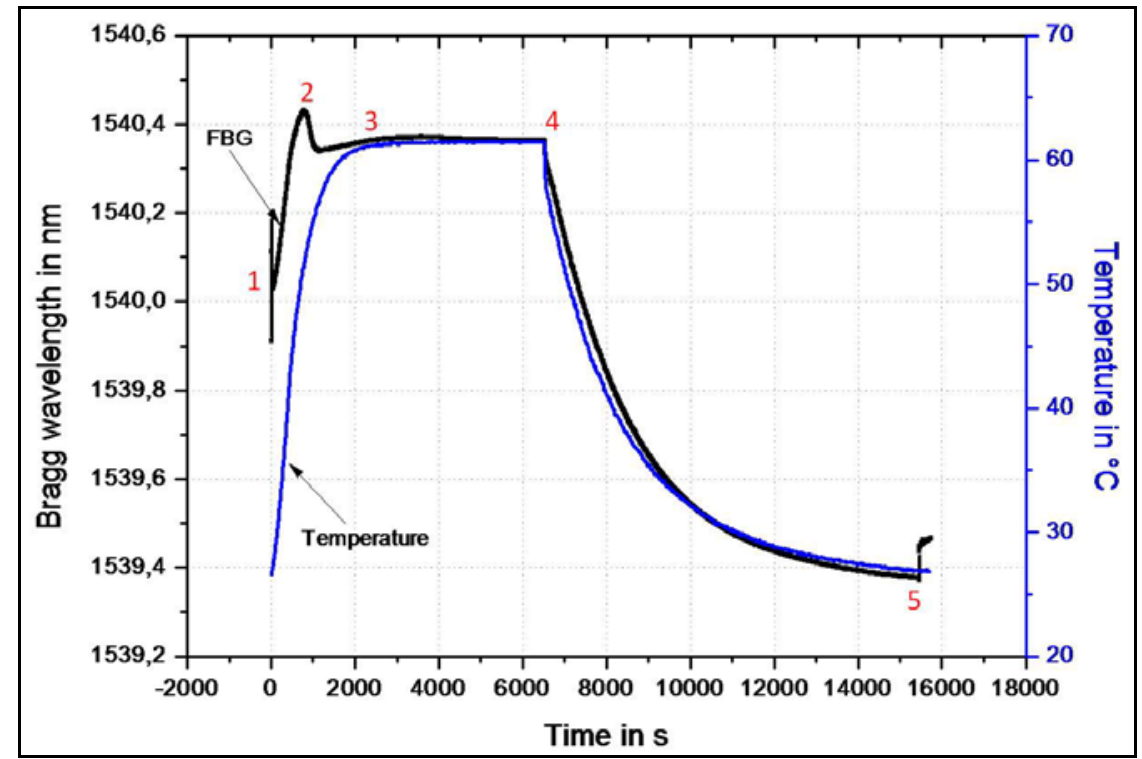

The total volume shrinkage (1-5) can be expressed as a change in the Bragg wavelength of the FBG. During heating, volume expansion (1-2) can be observed. Although the final curing temperature is not yet reached (2), the reaction shrinkage begins (2-3), which is observed in a significant drop in Bragg wavelength. After isothermal hardening (3-4), volume contraction due to cooling (4-5) is observed.

The embedded FBG was not temperature-compensated. The effects observed here cannot therefore clearly be separated into temperature load and mechanical forces acting at the sensor. For a description of the curing process, and for the use of these sensors for cure monitoring, it is necessary to develop a specially adapted sensor system based on fundamental work in literature [5-7]. Additionally, interferences on spectral phenomena have to be investigated, e.g. transverse forces and residual stresses.

\subsection{Interface Adhesion}

The characterization of interfacial interaction between the surrounding matrix and the sensor is of fundamental importance for a reliable sensor use. Therefore, the adhesion between the FBG and the patch carrier was investigated by using the single fiber push-out method. The fibers investigated with this method had different coatings as polyimide, Ormocer ${ }^{\circledR}$, and acrylate. Uncoated fibers were investigated, too.

The arrangement around the integrated fiber was evaluated by optical investigations of polished micrograph sections (Figure 4).

In Figure 4, it can be seen that the thick and relatively soft acrylate coating was significantly deformed during the manufacturing process of the patch. It was assumed that during the curing process, the surrounding matrix shrinks and the soft acrylate is pressed to the sides due to residual stresses developed in the material. Furthermore, the glass fiber cladding does not stick properly to the coating. In case of external forces acting at the patch, deformations take place and the fiber may totally lose its contact to the coating and may freely move or slip inside of it like in a tube. In this case of 
debonding, the specimen and patch deformation, and thus the forces, can no longer be transferred into the sensitive Bragg grating.

However, in case of bare fibers embedded in patches, a good adhesion between the fiber and the surrounding matrix could be observed (Figure 5).

Figure 4. Micrograph section of an embedded acrylate-coated optical fiber. In this particular case, the fiber is delaminated from its coating.

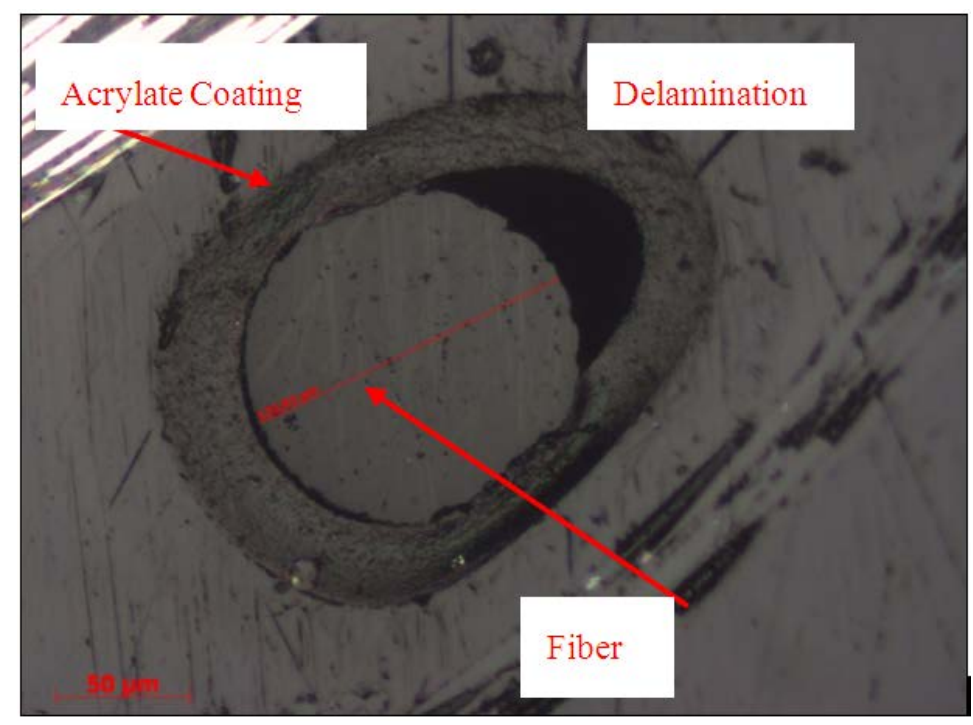

Figure 5. Embedded bare fiber without coating. The fiber has an excellent adhesion to the matrix.

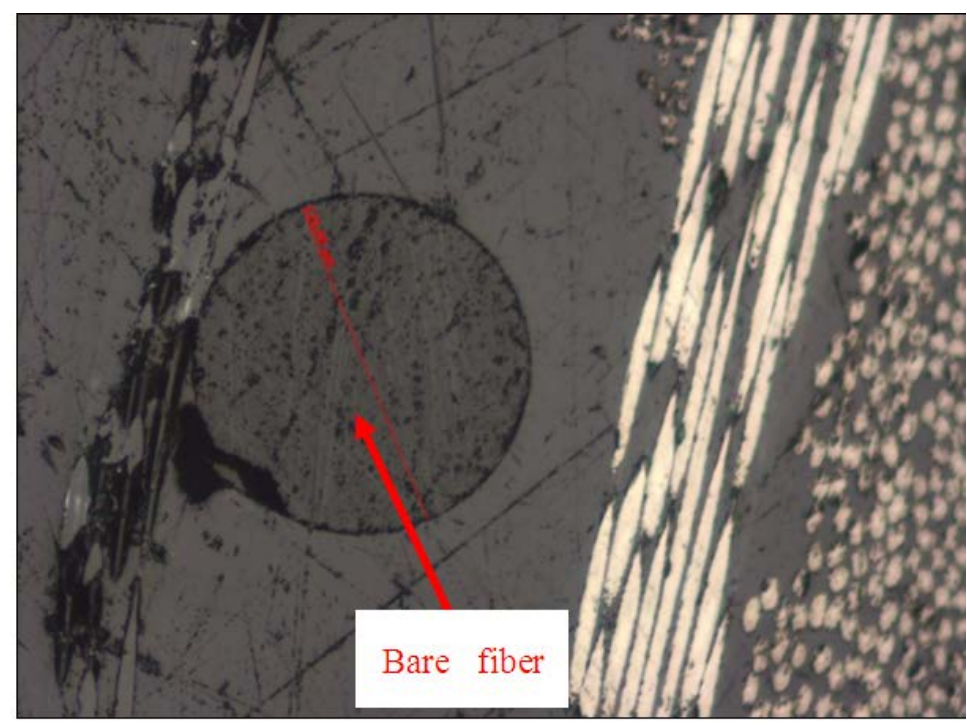

The bare fiber shows perfect connection to the surrounding matrix. Since the bare glass fiber is relatively stiff compared to a soft acrylate coating, it is therefore able to take the forces built up during curing and shrinking without large deformations. In the case of bare fibers, the shrinkage builds up forces pressing, even more so, the surrounding epoxy resin and the bare fiber together, leading to a good connection, even under external forces. Thus, external strain can directly be transferred into the optical fiber without additional deformations of the soft coating between the fiber and the matrix. 
For push-out tests, the polished micrograph sections were prepared as $500 \mu \mathrm{m}$ thick slices. A flat cone indenter with a diameter of $100 \mu \mathrm{m}$ was used to push the fiber out of this slice specimen. During the test, force and the indenter displacement were recorded. The shear stress acting at the fiber surface was calculated from the recorded force data and contact area. According to the optical observations under the microscope, the acrylate-coated fiber showed the worst adhesion, while the embedded bare fiber showed the best adhesion (Figure 6a,b).

Figure 6. Results of the indentation tests. The bare fiber (a) is able to bear about 50 times more stress than the glass fiber with the acrylate coating (b).

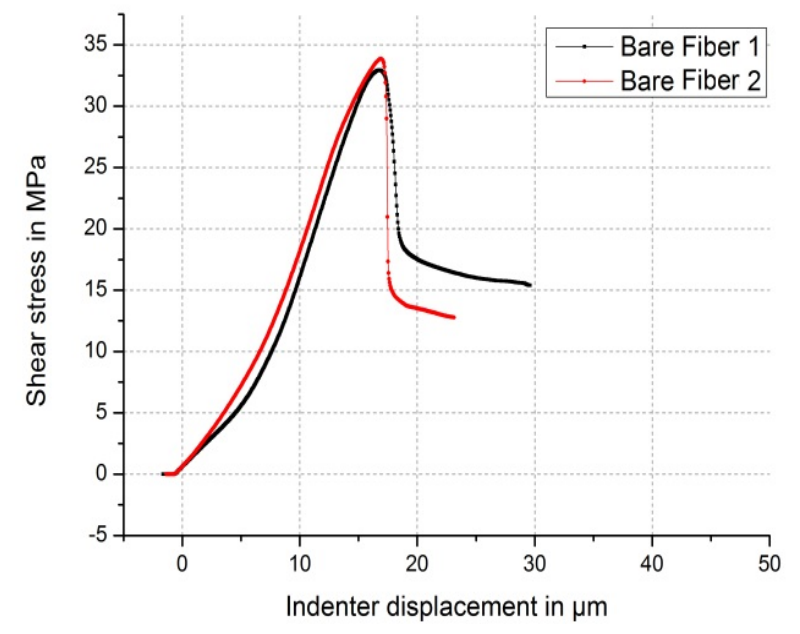

(a)

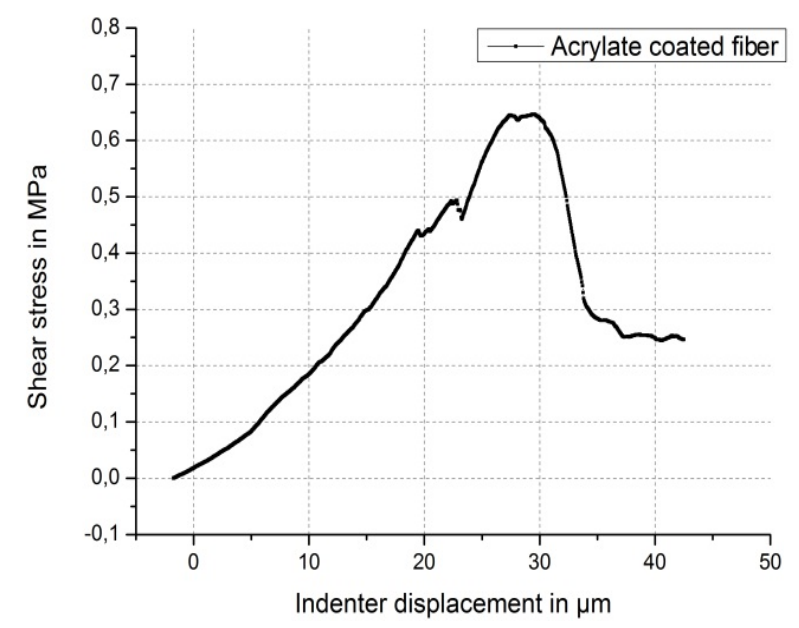

(b)

As shown in Figure 6, the maximum shear stress represents the estimated interfacial strength. For the embedded bare fiber (Figure 6a), it is approximately $34 \mathrm{MPa}$. The acrylate-coated fiber (Figure 6b) can easily be pushed out of the coating. Thus, the maximum shear stress is only 0.65 MPa. The frictional stress for bare and completely debonded fibers was evaluated as in between $17 \mu \mathrm{m}$ to $19 \mu \mathrm{m}$ indenter displacement, and is between $15 \mathrm{MPa}$ and $18 \mathrm{MPa}$. The frictional stress of the debonded acrylate-coated fiber was evaluated between $30 \mu \mathrm{m}$ and $34 \mu \mathrm{m}$ and is $0.25 \mathrm{MPa}$. It can be concluded, then, that the low shear strength between the glass fiber and the coating prevents the transfer of strain into the FBG, even when the thick and soft acrylate coating may prevent the FBG from strong lateral forces and local stress concentrations.

The obtained results from the push-out test for two other coating types, polyimide and Ormocer ${ }^{\circledR}$, are shown in Figure 7a,b.

The interfacial shear strength for Ormocer ${ }^{\circledR}$-coated fibers is around $20 \mathrm{MPa}$ and for polyimide-coated fibers about 13 . The better shear strength of these fibers compared to the acrylate coating could be explained by both, the better adhesion between glass fiber and coating, and the stiff coating material. The linear region for Ormocer ${ }^{\circledR}$-coated fibers (Figure $7 \mathrm{a}$ ) is at an indenter displacement of $1 \mu \mathrm{m}$ to $10 \mu \mathrm{m}$, which corresponds to $20 \mathrm{~mm} / \mathrm{m}$ to $24 \mathrm{~mm} / \mathrm{m}$ strain. Starting with $8 \mu \mathrm{m}$ indenter displacement, the coating seems to deform plastically. The fiber is finally detached from the coating at about $20 \mu \mathrm{m}$. 
Figure 7. Results of push-out tests on Ormocer ${ }^{\circledR}$ (a) and polyimide (b) coated fibers. Polyimide-coated fibers and Ormocer ${ }^{\circledR}$-coated fibers have relatively high adhesion to the matrix.

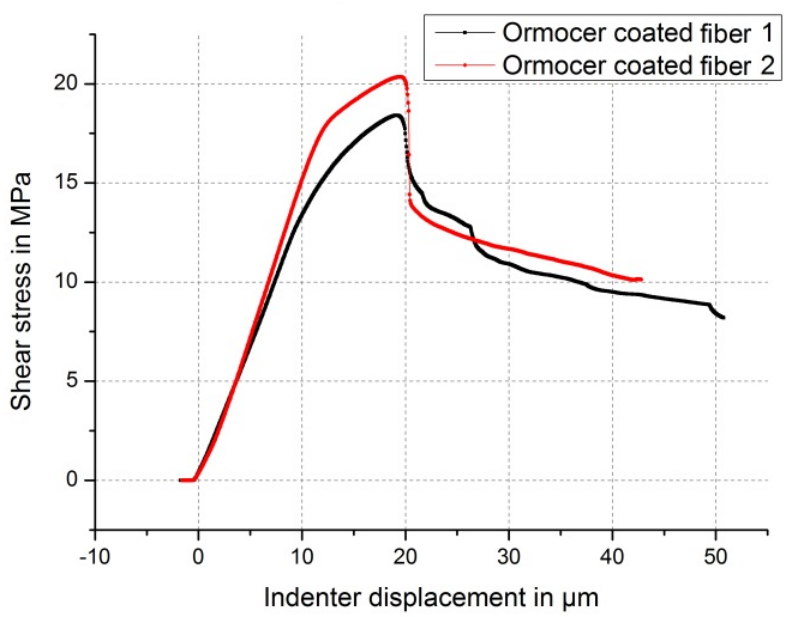

(a)

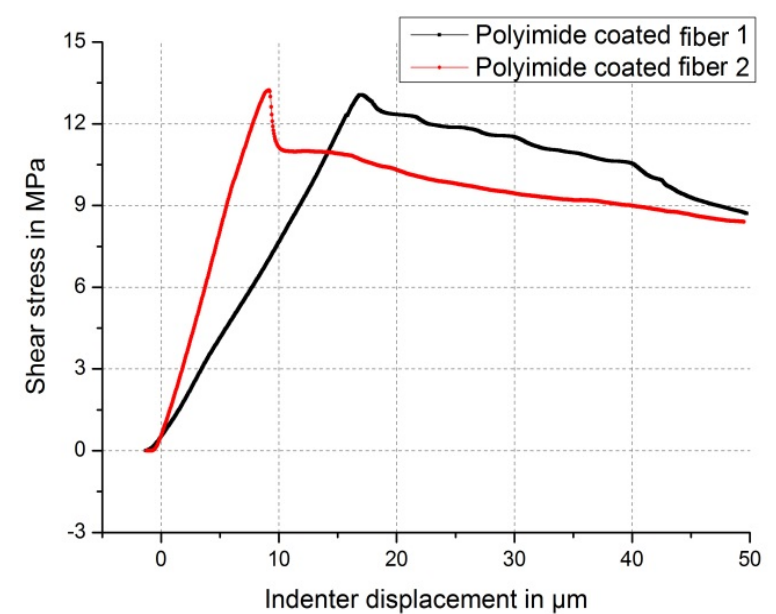

(b)

The force peak to overcome the interfacial adhesion of polyimide-coated fibers is not as high as for bare fibers or Ormocer ${ }^{\circledR}$-coated fibers, but an almost linear behavior can be observed for an indenter displacement up to $10 \mu \mathrm{m}$. With higher displacements, the fiber is apparently detached, and only friction takes place. In contrast to Ormocer ${ }^{\circledR}$-coated fibers, no plastic deformation could be observed.

Concluding the push-out tests, bare fibers show the best result in interface adhesion. However, when it comes to practical use, a coating for the fibers is required. Comparing coated fibers, polyimide has the best properties, followed by Ormocer ${ }^{\circledR}$.

\subsection{Application Technique}

The application of the patch to the specimen is the most common source of error concerning the strain transfer. As good as a patch may be designed, if the patch is not properly applied to the specimen, it cannot determine the correct strain of the specimen. The application mainly depends on two things: first, the choice of an adhesive well adapted to the measurement conditions and, second, the correct application. In this case, we decided to use Araldite 2020 and Z70 for the application of the patches to steel, aluminum and gfrp specimen, and we used Araldite 2015 and Z70 for cfrp specimen. Z70 is a very common adhesive for the application of resistance strain gauges, so we used that for comparison reasons. The Araldite adhesives have been recommended, especially for use with composite material.

In order to apply the sensing patch with optimum adhesion, a thin layer of adhesive was spread onto the patch and then it was pressed on to the specimen. The Araldite adhesives are hot-curing materials. The specimen with the patch was therefore packaged in a vacuum bag and heated up. Due to the uniform pressure inside the vacuum bag and the elevated temperature, the adhesive could spread homogeneously under the patch, and the patch adhered evenly to the specimen. A uniform, thin adhesive layer was achieved. Sharp edges that result from inhomogeneous spread of the adhesive could be avoided. With this method, the adhesive layer, as shown in Figure 2, slowly rises from the specimen 
to the edge of the patch so that shear forces develop slowly. This is a significant pre-condition for high dynamic loadings. If the shear forces abruptly change, this may possibly initiate delamination at the sensor-specimen interface.

\subsection{Temperature Sensitivity}

After developing and designing the patch, its characteristics were determined. That means the patch responses to temperature, load, combined temperature and load, and dynamic load were investigated. The patch responses to these parameters describe the patch characteristics. A total number of 13 patches were designed to carry out the different tests.

Before loading the patch, the effect of thermal influence on the patch was characterized. It is very important to determine this parameter. Typically, FBG sensors measure both temperature and strain. In order to achieve a pure strain measurement, the measured values obtained must be corrected by the temperature-correlated strain.

The temperature tests were carried out with patches with embedded acrylate-coated FBGs; the samples were investigated in a temperature chamber in the range of $-40{ }^{\circ} \mathrm{C}$ to $+60{ }^{\circ} \mathrm{C}$. The patches showed linear temperature sensitivity over the whole temperature range. The temperature sensitivity could be determined to be $24 \mathrm{pm} / \mathrm{K}$.

Temperature compensation during mechanical loading has been performed utilizing an additional temperature sensor.

\subsection{Strain Gauge Factor}

In conformity to the guideline for strain sensors based on fiber Bragg gratings [3], the patches were analyzed concerning their strain gauge factor and the temperature dependence of the strain gauge factor.

Previous investigations have shown that there are quite large disagreements between specifications of the manufacturers of commercially available fiber Bragg grating patches and the observed characteristics [2]. The strain gauge factor and the temperature dependence of the strain gauge factor of the designed patches were therefore characterized. The patches with embedded acrylate-coated FBGs were applied together with resistance strain gauges on stainless steel and aluminum tensile test coupons. The resistance strain gauges were chosen for referencing the strain gauge factor of the patches, while the coupons were only statically loaded with relative small loads (up to $2000 \mu \mathrm{m} / \mathrm{m}$ ).

At first, the tensile test coupons were loaded up to a maximum load of $15 \mathrm{kN}$ in three cycles to pre-stress the coupons. After that, the coupons were loaded in steps of $1.5 \mathrm{kN}$ up to the maximum load, and down again also in three cycles. The maximum load of $15 \mathrm{kN}$ correlates to a strain of $740 \mu \mathrm{m} / \mathrm{m}$ of the stainless steel coupons and a strain of $1800 \mu \mathrm{m} / \mathrm{m}$ of the aluminum coupons. During the whole test, the temperature was kept constant at $23{ }^{\circ} \mathrm{C}$. All the investigated patches showed linear strain behavior over the total loading cycle, as shown in Figure 8. The strain gauge factor was determined to be 0.7799 . 
Figure 8. Determination of the strain gauge factor from the change in Bragg wavelength compared to resistance strain gauges.

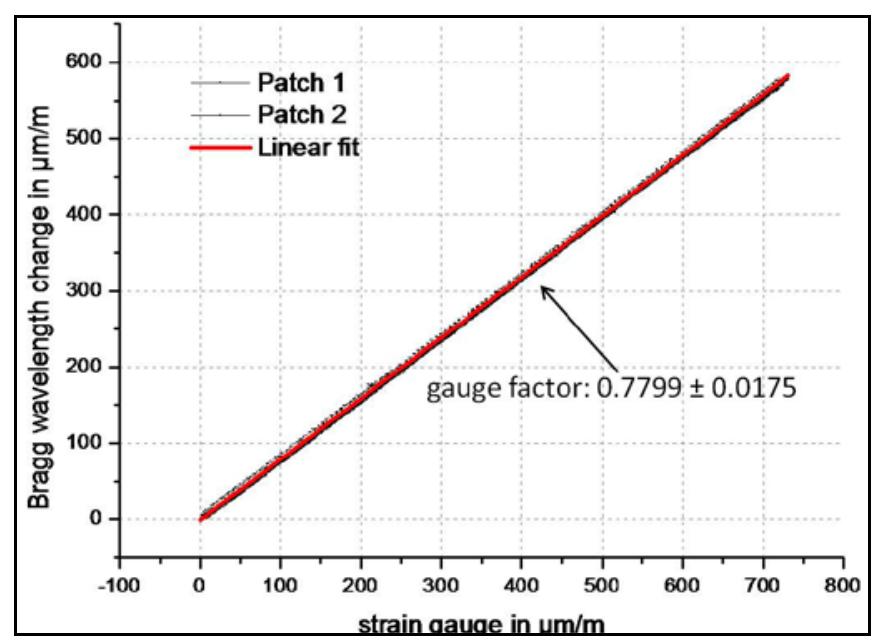

Figure 9. Change in Bragg wavelength of a patch compared to the strain determined with resistance strain gauges at different temperatures.

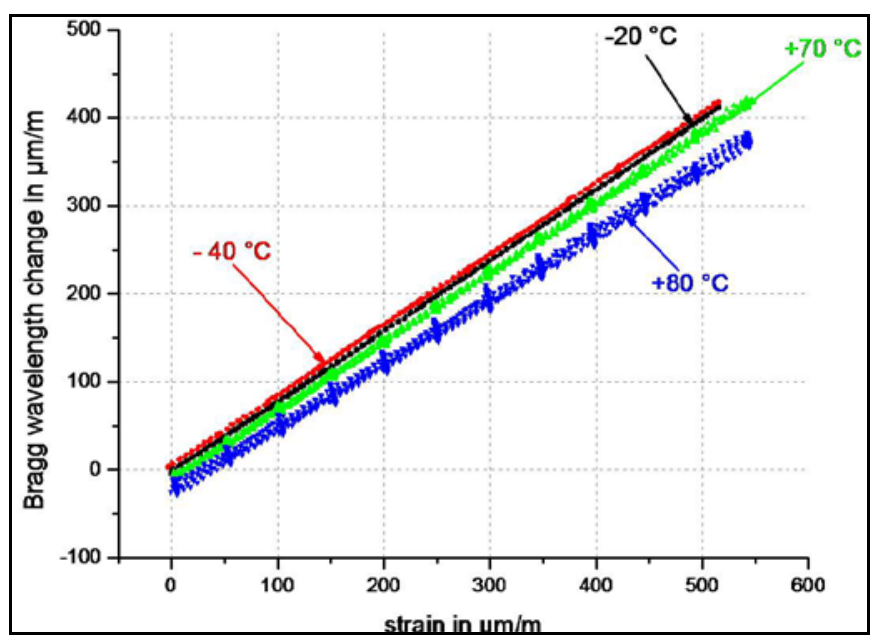

To analyze the temperature dependence of the strain gauge factor, the tensile test coupons were loaded in the same way as for the tests to determine the strain gauge factor at room temperature. These tests were carried out at temperatures of $-40^{\circ} \mathrm{C},-20^{\circ} \mathrm{C}, 70^{\circ} \mathrm{C}$ and $80^{\circ} \mathrm{C}$. Although the patches were manufactured at a curing temperature of $60{ }^{\circ} \mathrm{C}$, it was necessary to determine how and under what temperature and load the patches start to degrade. Before starting with the test, the patches were post-cured at a temperature of $82{ }^{\circ} \mathrm{C}$. The loading cycles started when the temperature chamber reached thermal equilibrium.

In Figure 9, it can be observed how the strain gauge factor varies with temperature. Between $-40{ }^{\circ} \mathrm{C}$ and $+70{ }^{\circ} \mathrm{C}$, the strain gauge factor is stable. At $+80{ }^{\circ} \mathrm{C}$, the strain gauge factor amounts to $0.6896 \pm 0.0451$. The strain gauge factor dropped nearly $12 \%$ compared to the strain gauge factor at room temperature. Additionally, it can be observed that at higher temperatures, the single data points spread farther from the ideal linear fit curve than at lower temperatures. This is probably due to an initiation of material changes of the patch carrier near the post-curing temperature. 
In case of all other temperatures, the strain gauge factor is in a reasonable range, as also determined for room temperature. This can be concluded from Table 1.

Table 1. Strain gauge factors determined from combined temperature and loading cycles.

\begin{tabular}{cccccc}
\hline Temperature & $-\mathbf{4 0}{ }^{\circ} \mathbf{C}$ & $-\mathbf{2 0}{ }^{\circ} \mathbf{C}$ & $\mathbf{2 3}^{\circ} \mathbf{C}$ & $\mathbf{7 0}^{\circ} \mathbf{C}$ & $\mathbf{8 0}{ }^{\circ} \mathbf{C}$ \\
\hline Gauge factor & $0.7955 \pm 0.0081$ & $0.7937 \pm 0.0103$ & $0.7799 \pm 0.0175$ & $0.7805 \pm 0.0081$ & $0.6896 \pm 0.0451$ \\
\hline
\end{tabular}

It can be concluded from these experiments that the developed patches with embedded acrylate-coated FBGs show a linear and constant strain behavior over a temperature range of $-40{ }^{\circ} \mathrm{C}$ to $+70{ }^{\circ} \mathrm{C}$, if a post-curing has been carried out over $80^{\circ} \mathrm{C}$. The strain gauge factor is slightly temperature dependent, but the corresponding gauge factor error over the whole temperature range is $1.4 \times 10^{-4} 1 / \mathrm{K}$. It is assumed that this effect is not significant in this temperature range, and for practical applications, the average strain gauge factor for the designed patches is calculated to be $0.7874 \pm 0.0081$. The relative error of the gauge factor is about $1 \%$ and therefore comparable to the gauge factor error of established resistance strain gauges.

\subsection{Spectral Phenomena}

During static testing of the patches, the reflected small spectrum was analyzed and characterized to determine major changes in the spectrum design. A deformation of the spectrum, together with growing side lopes at higher loads, can lead to misinterpretations of the Bragg wavelength. If the side lopes are as high as the main peak, or the main peak starts to broaden extremely, the mathematical algorithm used by the interrogator system cannot distinguish between several peaks and, consequently, is not able to accurately define the Bragg wavelength. In order to interpret the reflected signal correctly, a side lope suppression of at least $5 \mathrm{~dB}$ under the Bragg peak is necessary, as defined in the VDI guideline [3]. The reflected spectra at each load step for a patch with embedded bare fiber is shown in Figure 10.

Figure 10. Reflected spectrum of the FBG patch with embedded bare fiber at different load steps.

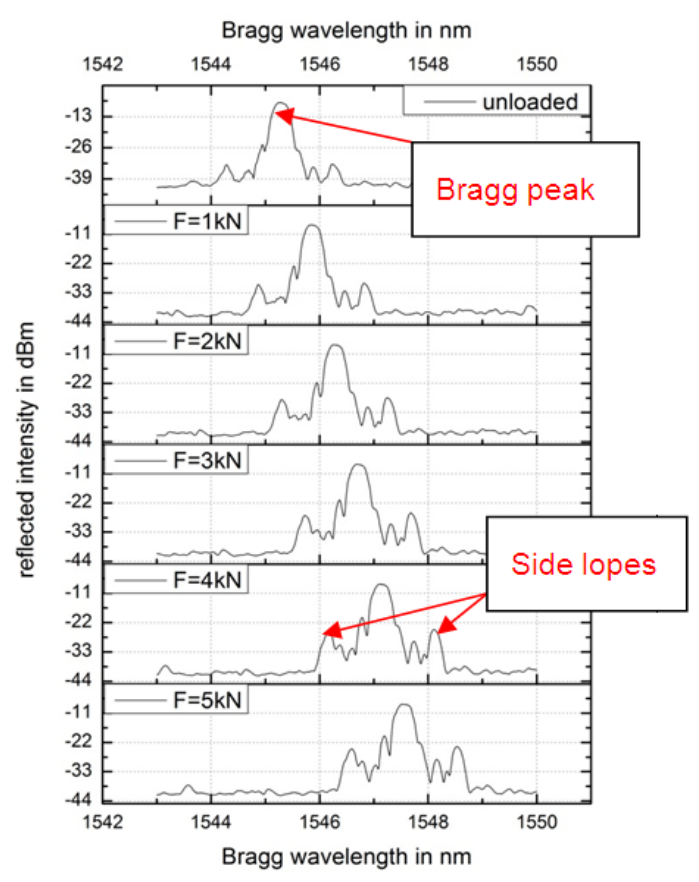


A maximum load of $5 \mathrm{kN}$ is equivalent to a strain of approximately $1500 \mu \mathrm{m} / \mathrm{m}$. In order to interpret the spectral phenomena, a patch with embedded bare fiber was chosen. In this way, transverse force effects that directly act onto the bare fiber without a protecting coating could clearly be studied.

Figure 11 reveals that, indeed, side lopes rise equally to the left and right of the Bragg peak during the different loading steps, but the Bragg peak itself does not broaden significantly. Presumably, there are no transverse forces acting on the grating. It is assumed that the side lopes were a result of the patch manufacturing process. During curing, the grating is likely partly pre-strained due to small local residual stresses. This may lead to a partially chirped embedded grating. When starting the patch loading, the grating is stretched non-uniformly due to initial chirp. This may lead to the increase of the side lopes. However, the increasing side lopes do not affect the functionality of the patches. The side lope suppression is significantly $13 \mathrm{~dB}$ below the main Bragg peak, and fulfills, therefore, the requirements of the VDI strain sensor guideline [3].

The spectral effects of a combination of longitudinal and transverse load and local strain gradients on the embedded FBG, like it is the case in a multi-axial strain field, is of a very complex nature and has not been focused in this context.

\subsection{Fatigue Behavior}

The fatigue behavior of the patches and the error resulting due to fatigue effects have to be characterized in order to utilize the patches, not only for measurements of statically loaded structural elements, but also for dynamically loaded ones. Important parameters are the maximum dynamic load that the patch is able to withstand and the corresponding strength of the adhesive bond between patch and specimen. A spectral analysis was not in the focus for the fatigue tests. Since practical users are usually only interested in strain information or, at most, the Bragg wavelength change, spectral changes due to perturbation effects have not been considered as long as the measurement unit could identify the Bragg peak.

Referencing with resistance strain gauges is not suitable in the case of dynamic loads, because resistance strain gauges are limited in their fatigue life. Furthermore, resistance strain gauges are designed for measurements of classical materials like steel or concrete. For highly tensile materials like cfrp or gfrp, it is necessary to use appropriate referencing techniques. Most common are optical reference techniques that scan the surface of the specimen, like digital image correlation (DIC) or laser extensometer. In this case, a laser extensometer was chosen as the reference method.

Four patches were used in tension fatigue tests. Figure 11 shows the set-up of the tensile fatigue test. The patches were applied to cfrp tensile test coupons and strained in 10 steps up to $10 \mathrm{~mm} / \mathrm{m}$. Each step consisted of 10,000 load cycles carried out with a frequency of $3 \mathrm{~Hz}$. The tension fatigue test was used to determine the maximum strain that the patch can withstand. This parameter was then afterwards used to set up a dynamic fatigue test.

Half of the analyzed patches had embedded FBGs with acrylate coating; the others were embedded as bare fibers. It became evident during the test that the patches with integrated acrylate-coated FBG endured the tensile fatigue test until the specimen broke (Figure 12). In Figure 13, the strain measured with the patch for maximum and minimum load is shown together with the measured strain of the laser extensometer. The specimen broke after 111,890 loading cycles. The broken specimen revealed that 
the patch started to debond at its edges. Also small cracks in the adhesive were observed. This behavior is considered as a first indication for a potential failure of the patch itself.

Figure 11. Set-up of the fatigue test. The patches are applied to the back and front side of a cfrp specimen. A laser extensometer is used to reference the strain values at the small side of the specimen.

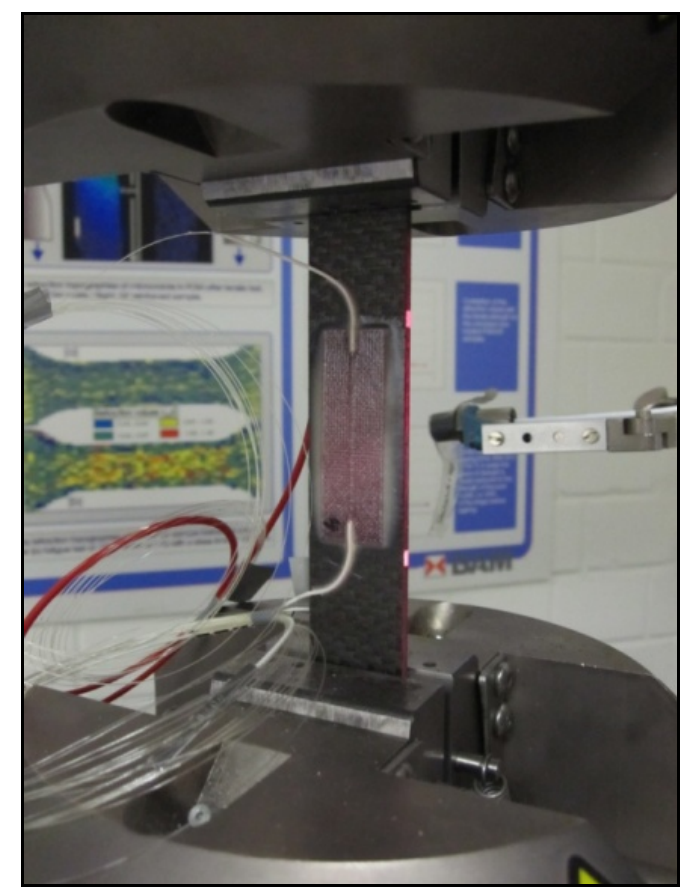

Figure 12. Broken specimen after tensile fatigue test. The crack initiation zone lies under the patch, but the patch did not peel off from the surface of the specimen.

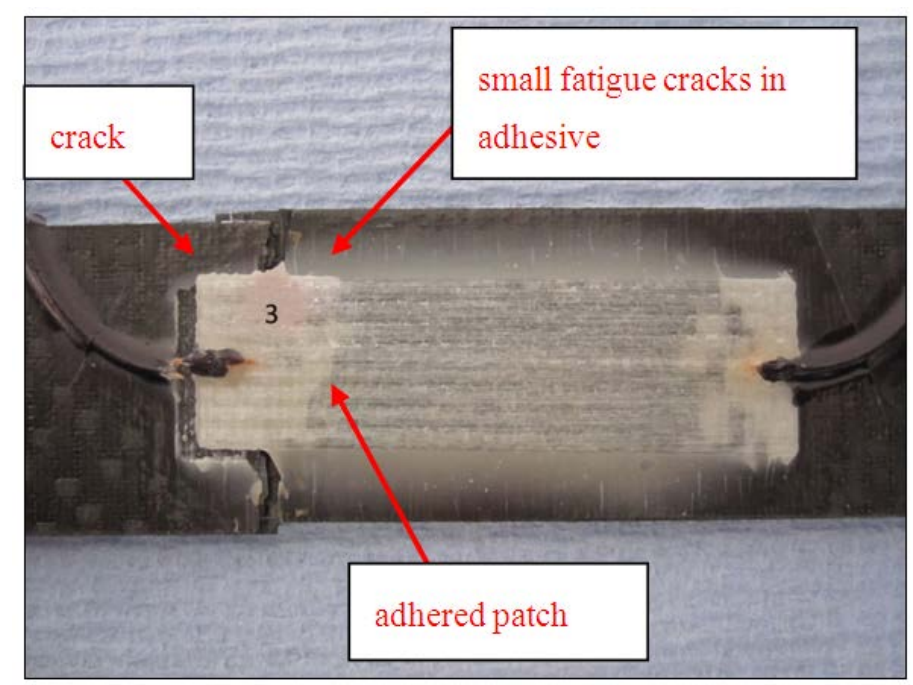


Figure 13. Strain response of a patch with embedded acrylate-coated FBG compared to the strain response of the laser extensometer for maximum and minimum load.

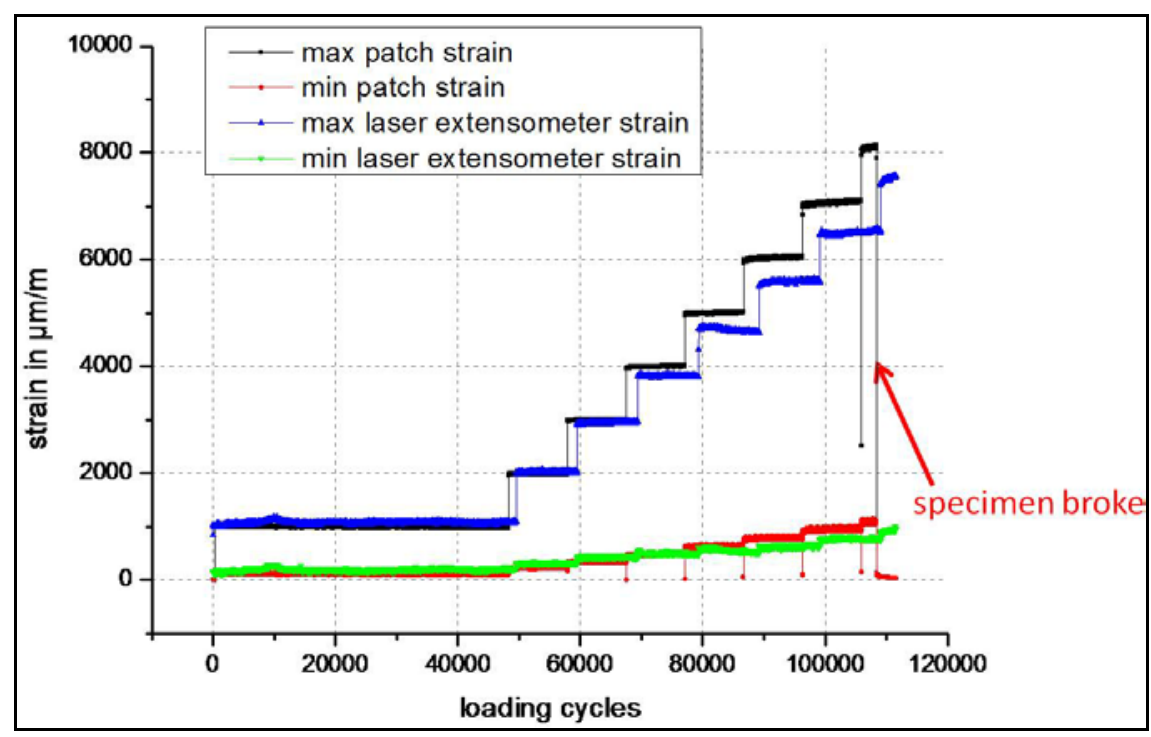

The difference in strain measured with the patch and with the laser extensometer, as observed in Figure 13, can probably be explained by a change in the gauge factor at higher loads. As observed during indentation tests, the embedded acrylate fiber shows delamination between glass fiber and acrylate coating. With increasing loads, the adhesion between glass cladding/core and coating/matrix decreases, and, consequently, the gauge factor declines. The error due to a change in gauge factor is significant in the case of embedded acrylate-coated fibers, and leads to misinterpretation of the measured patch response at higher dynamic loads, and, consequently, to an error in the specimen strain of about $10 \%$. Because of the extremely bad performance, it was decided not to continue the investigations with patches containing embedded acrylate-coated FBG.

The attached patches with integrated bare fibers, on the contrary, failed before the specimen broke (Figure 14). This graph shows the minimum and maximum strain during loading cycles of the patches and the laser extensometer. It could clearly be seen that both patches failed shortly one after the other after around 80,000 loading cycles. Although embedded bare fibers reach the best interface adhesion with the surrounding matrix and show an excellent gauge factor during static loading tests, during dynamic loading they show a limited fatigue life. The reason for failure of the patches is not a bad application. The patches still bonded perfectly even after failure. It was observed that the bare fiber broke inside the patch. Part of the coated fiber with an outer diameter of $250 \mu \mathrm{m}$ goes inside of the patch, and the coating was removed for a length of $25 \mathrm{~mm}$ so that the bare fiber with the sensitive FBG, outer diameter $125 \mu \mathrm{m}$, lies inside of the patch. The change in diameter from coating to bare fiber is very abrupt, so that the internal stress of the fiber concentrates at the point of diameter change (see Figure 15). This leads to a fiber breakage exactly at the points of stress concentration, so that the patch fails long before the specimen cracks. 
Figure 14. Two patches with embedded bare FBG were applied on the front and back of a specimen and loaded during tensile fatigue tests. Both patches failed clearly before reaching beginning crack initiation of the specimen.

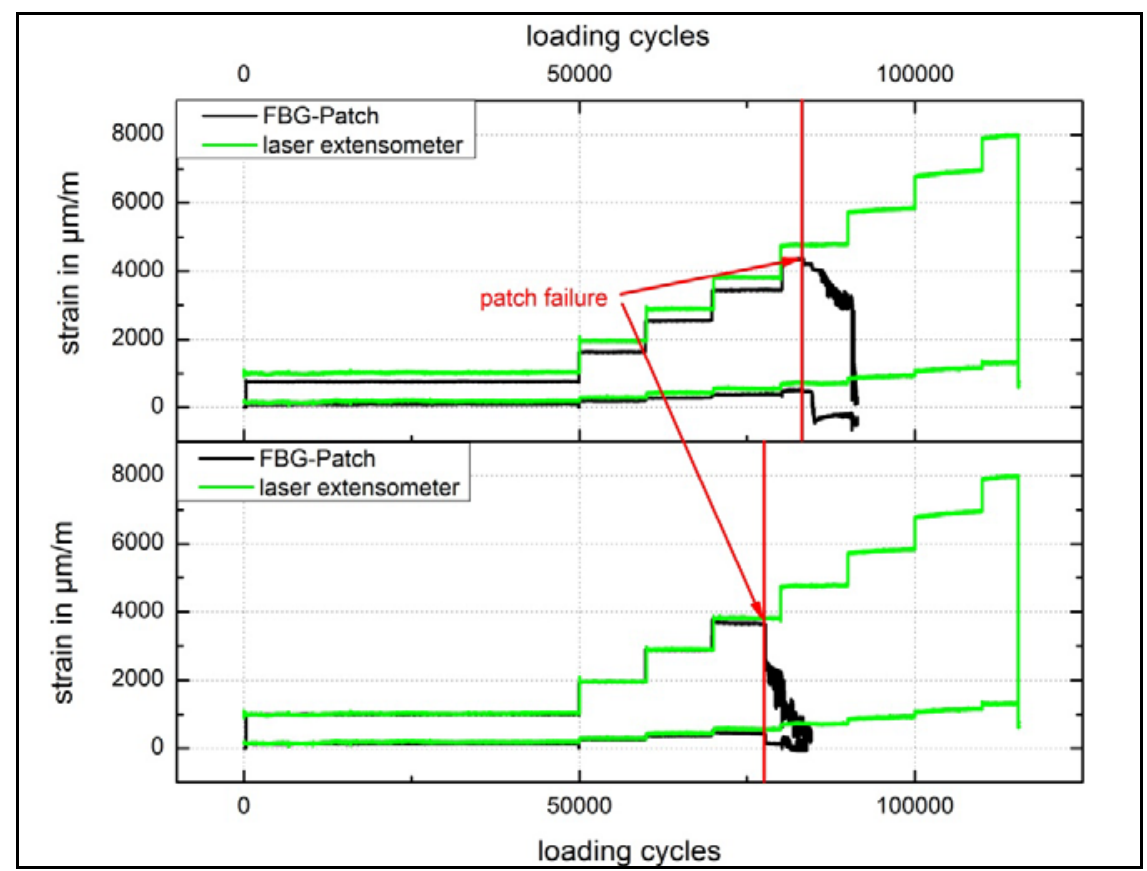

Figure 15. Schematic drawing of the stress concentrations of the embedded fiber inside a patch under dynamic loading.

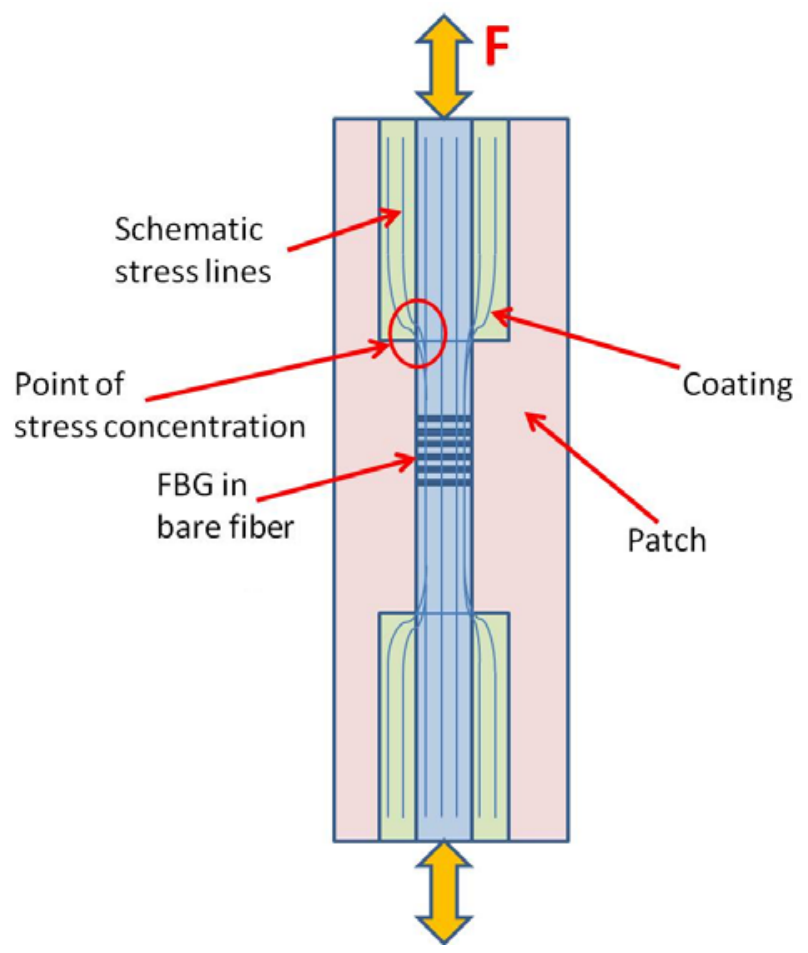

Therefore, it was decided to use patches with embedded Ormocer ${ }^{\circledR}$-coated fibers to avoid early failure of the patch during dynamic fatigue tests. Ormocer ${ }^{\circledR}$-coated fibers showed good interfacial adhesion and a relative good strain gauge factor. 
The parameters for the dynamic fatigue test were chosen according to the experimentally determined strain parameters from the tensile fatigue tests. The test was force-controlled. The tests were carried out with a frequency of $3 \mathrm{~Hz}$ and an alternating load between $2 \mathrm{kN}$ and $20 \mathrm{kN}$. The loading was increased stepwise every 10,000 cycles. The maximum load corresponded to a theoretical strain of $6000 \mu \mathrm{m} / \mathrm{m}$. In Figure 16, the strain of the patch with an embedded Ormocer ${ }^{\circledR}$-coated fiber and the strain of the referencing laser extensometer are shown for maximum and minimum load.

Figure 16. Dynamic fatigue test of a FBG patch referenced with laser extensometer.

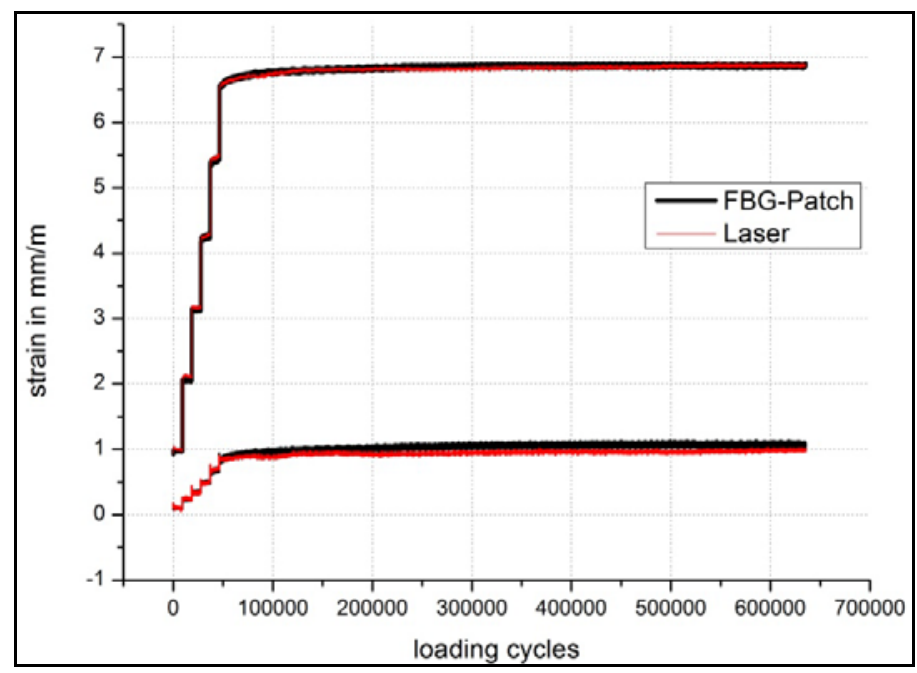

Obviously, the patch is able to withstand up to 650,000 loading cycles without failure. It is assumed that the patch would bear up to 1,000,000 loading cycles, but this has to be proved yet. It can also be seen from Figure 16 that the strain of the specimen measured with both the patch and the laser extensometer increases over the whole load cycling. This behavior probably results from a beginning plastic material deformation that leads to a change in the Young's modulus of the specimen.

\section{Conclusions}

Large deviations in the specifications of commercially available patches from their real characteristics motivated us to develop a special field deployable FBG patch for applications in structural health monitoring systems. The designed patch was characterized and improved stepwise, and showed excellent long-term stability in dynamic fatigue tests. Compared to commercially available patches, the developed patch completely uses gfrp carrier material.

Depending on the measurement task, either static or dynamic, it is necessary to decide which fiber type should be integrated in the patches. Bare fibers show best results in strain transfer and can be used for static load measurements. For dynamic loadings, the fiber has to be coated either with polyimide or Ormocer ${ }^{\circledR}$ to withstand the high dynamic loadings without failure. Acrylate-coated fibers are not recommended for embedment into patches because of their bad interface adhesion and the erroneous gauge factor during fatigue tests.

During the basic research to develop and design gfrp patches with embedded FBG sensors, a great deal of essential experience and skills could be obtained. This enormous growth in knowledge can be used for adaptation of FBG sensors and their integration in any composite structures. Additional 
knowledge concerning temperature sensitivity, strain transfer characteristics and gauge factor, as well as fatigue behavior for a reliable integration into composite structures, can be derived from the described basic research work.

\section{Acknowledgments}

The authors would like to acknowledge the assistance provided by the BAM group "Fatigue testing, failure analysis, damage mechanics” in carrying out several fatigue tests.

\section{References}

1. Othonos, A.; Kalli, K. Fiber Bragg Gratings-Fundamentals and Applications in Telecommunications and Sensing; Artech House: Bosten \& London, MA, USA, 1999; pp. 96-98.

2. Schlüter, V.G.; Kusche, N.; Habel, W.R. How Reliably do Fibre Bragg Grating Patches Perform as Strain Sensors? Presented at EWOFS 2010-4th European Workshop on Optical Fibre Sensors, Porto, Portugal, 8-10 September 2010.

3. VDI/VDE/GESA Guideline 2660, Experimental Stress Analysis-Optical Strain Sensor based on fibre Bragg grating; Basics, Characteristics and its Testing, 2010. Available online: http://www.beuth.de (accessed on 11 September 2012).

4. Wenzel, M. Spannungsbildung und Relaxationsverhalten bei der Aushärtung von Epoxidharzen (Tension formation and relaxation behavior of epoxy resins during curing process), Ph.D. Thesis, Technical University of Darmstadt, May 2005.

5. O’Dwyer, M.J.; Maistros, G.M.; James, S.W.; Tatam, R.P.; Partridge, I.K. Relating the state of cure to the real-time internal strain development in a curing composite using in-fibre Bragg gratings and dielectric sensors. J. Meas. Sci. Technol. 1998, 9, 1153-1158.

6. Antonucci, V.; Giordano, M.; Cusano, A.; Nasser, J.; Nicolais, L. Real time monitoring of cure and gelification of a thermoset matrix. J. Compos. Sci. Technol. 2006, 66, 3273-3280.

7. Hernández-Moreno, H.; Collombet, F.; Douchin, B.; Choqueuse, D.; Davies, P.; González Veláquez, J.L. Entire Life Time Monitoring of Filament Wound Composite Cylinders Using Bragg Grating Sensors: II. Process Monitoring. J. Appl. Compos. Mater. 2009, 16, 197-209.

(C) 2013 by the authors; licensee MDPI, Basel, Switzerland. This article is an open access article distributed under the terms and conditions of the Creative Commons Attribution license (http://creativecommons.org/licenses/by/3.0/). 